\title{
RADIATION HARD MONOLITHIC CMOS SENSORS WITH SMALL ELECTRODE SIZE FOR THE ATLAS EXPERIMENT IN THE HL-LHC
}

\author{
I. Asensi Tortajada ${ }^{1,2}$, M. Barbero3, I. Berdalovic ${ }^{1,4}$, S. Bhat ${ }^{3}$, D. Bortoletto5, C. Buttar ${ }^{6}$, \\ I. Caicedo7, R. Cardella",2, F. Dachs", ${ }^{1 *}$, V. Dao', Y. Degerli', M. Dyndal', L. Flores Sanz de Acedo, ${ }^{1,10}$, \\ P. Freeman"1 A. Habib ${ }^{4}$, T. Hemperek7, B. Hiti'12, T. Kugathasan', K. Moustakas ${ }^{7}$, M. Munker', \\ H. Pernegger', F. Piro', P. Riedler', E.J. Schioppa', P. Schwemling', A. Sharma',5, L. Simon Argemi' ${ }^{10}$, \\ W. Snoeys ${ }^{1}$, C. Solans Sanchez', T. Suligoj ${ }^{4}$, T. Wang ${ }^{7}$, N. Wermes ${ }^{7}$
}

\author{
${ }^{1} \mathrm{CERN}(\mathrm{CH})$ \\ ${ }^{2}$ University of Valencia CSIC (ES) \\ 3Centre de Physique des Particules Marseille (FR) \\ 4University of Zagreb (HR) \\ 5University of Oxford (UK) \\ ${ }^{6}$ University of Glasgow (UK) \\ 7University of Bonn (DE) \\ ${ }^{8}$ Vienna University of Technology (AT) \\ 9IRFU-CEA-Saclay (FR) \\ 10 University of Glasgow (UK) \\ ${ }^{11}$ University of Birmingham (UK) \\ ${ }^{12}$ Jozef Stefan Institute (SL)
}

\begin{abstract}
Mini-MALTA is a Monolithic Active Pixel Sensor prototype developed in the TowerJazz 180 nm CMOS imaging process, with a small collection electrode design (3um), and a small pixel size (36.4 um), on high resistivity substrates and large voltage bias. It targets the outermost layer of the ATLAS ITK Pixel detector for the HL-LHC. This design addresses the pixel in-efficiencies observed in MALTA and TJ-Monopix to meet the radiation hardness requirements. This contribution will present the results from characterisation in particle beam tests that show full efficiency up to 1 E15 neq/cm2 and 70 Mrad.
\end{abstract}

Keywords: CMOS, DMAPS, monolithic, pixel, radiation hard, silicon, HL-LHC, ATLAS, TowerJazZ

\section{INTRODUCTION}

The CERN ATLAS Pixel group explores a standard CMOS process as an option to produce monolithic silicon pixel detectors for the coming high luminosity upgrade of the LHC at CERN [1,2]. Specifically, the aim is to develop a pixel detector for the $5^{\text {th }}$ layer of the planned ATLAS Inner Tracker (ITk).

The increase in radiation damage, the much larger surface area of the tracker and the demand for improved tracking performance motivate the development of new pixel detectors that offer low material budget as well as easy and cheap production at large scale. The challenge is to develop such a sensor which can also maintain a sufficient particle detection efficiency of $>97.5 \%$ up to a total ionizing dose (TID) of $80 \mathrm{MRad}$ and a non-ionizing energy loss (NIEL) of $10^{15} 1 \mathrm{MeV}$ neutron equivalent fluence per $\mathrm{cm}^{2}\left(10^{15} 1 \mathrm{MeV} \mathrm{n}_{\mathrm{eq}} / \mathrm{cm}^{2}\right)$. A full spectrum of the expected charged hadron and neutron irradiation at the $5^{\text {th }}$ layer of the planned ATLAS ITk can be found in
[3]. While state-of-the-art pixel detector systems achieve this already, they do not offer the low material budget nor the powerful production infrastructure provided by a monolithic silicon pixel detector manufactured with the $180 \mathrm{~nm}$ process by TowerJazz. Initial tests were done in 2017 using a design with an additional planar n-doped layer in the epitaxial layer to achieve homogenous depletion across the whole pixel volume [4]. The exact doping profile of this layer is not disclosed by the foundry but was chosen to withstand the expected effects of donor/acceptor removal up to lifetime irradiation.

Following these tests, two large scale designs were fabricated. These designs, however, showed poor charge collection at the pixel borders [5,6]. Detailed TCAD simulations [7] showed that the low lateral electric field near the pixel borders significantly increases the collection time for the signal charge generated in that area, especially for larger pixel pitches. This in turn causes significant signal degradation for irradiated sensors.

\footnotetext{
*dachsf@gmail.com
} 
2. CMOS SILICON PIXEL SENSORS WITH SMALL COLLECTION DIODES

The chosen design for a CMOS manufactured Depleted Monolithic Active Pixel Sensor (DMAPS) features a small collection diode (3 microns diameter) and a $36.4 \times 36.4$ micron pixel pitch.

The small collection diode reduces capacitance and thus noise which allows low thresholds (down to 150 electrons) and thus to reduce the sensor thickness to 100 microns or even less. The main challenge for this pixel design is to maintain sufficient charge collection and thus efficiency after irradiation.

Beam tests with a first prototype "MALTA" (Monolithic from ALice To Atlas) showed significant efficiency losses at the pixel borders where the deposited charge is farthest away from the collection diode after irradiation with reactor neutrons to $10^{15} 1$ $\mathrm{MeV} \mathrm{n}_{\text {eq }} / \mathrm{cm}^{2}$. Deposited charge is not collected fast enough and signal degradation due to charge trapping sets in (see Fig. 1).

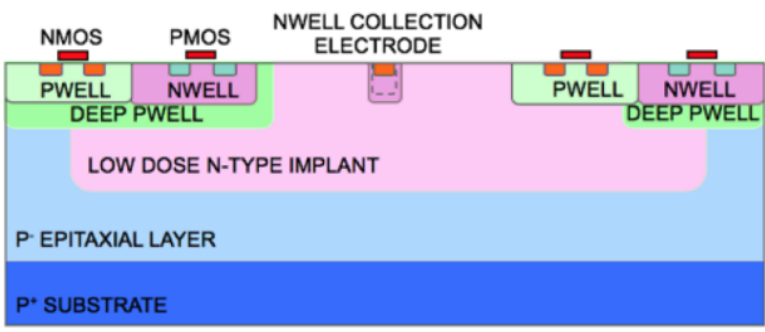

Figure 1. Improved pixel design with n-gap [8]

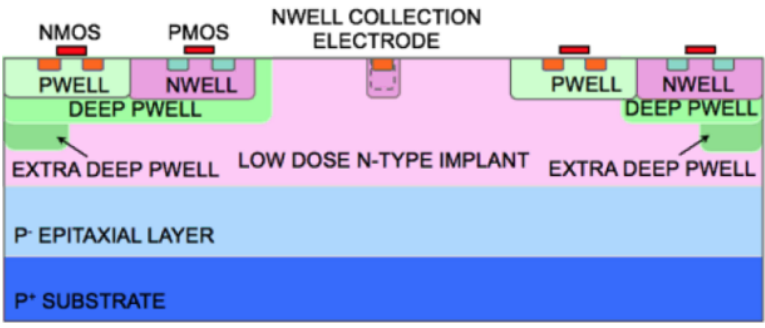

Figure 2. Improved pixel design with extended p-well [8]

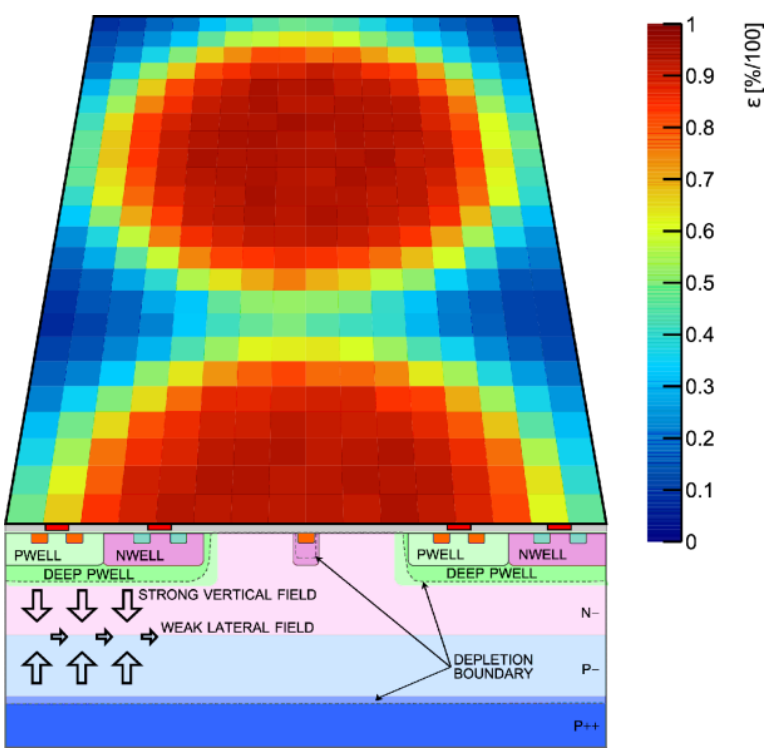

Figure 3. Sketch of the efficiency drop at Pixel borders for small collection diode designs. The weak lateral field between Pixels causes slow charge collection
Results from following TCAD simulations prompted two possible solutions to address these issues. The first approach is to extend the deep p-well, which shields PMOS transistors from the collection diode, deeper into the epitaxial layer between pixels ("extended p-well", see Fig. 2). The second approach was to remove the planar n-doped implantation of the epitaxial layer between pixels ("n-gap", see Fig. 3). Both approaches result in a more positive space charge in the epitaxial layer between pixels, which improves the lateral electric field component and thus charge collection. Both approaches were implemented in a new chip design "Mini-MALTA".

\section{THE MINI-MALTA SENSOR}

The Mini-MALTA sensor features a $16 \times 64$ square pixel matrix with a 36.4 micron pitch. The matrix is divided into 8 sectors with varying designs for the pixel and its analogue front-end. An overview of the features of these sections is given in Table 1. An image of the sensor can be seen in Fig. 4 .

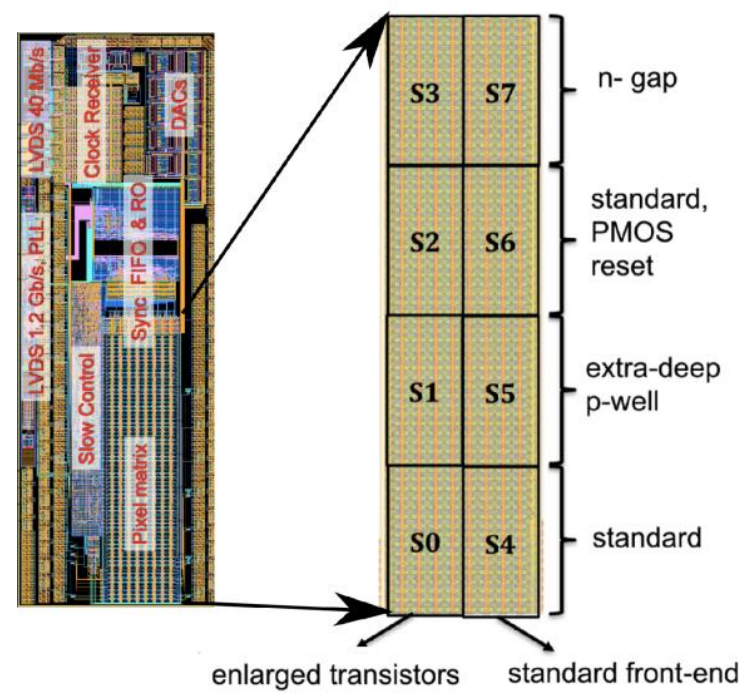

Figure 4. Mini-Malta chip and matrix layout [8]

Table 1. Mini-Malta matrix sectors [8]

\begin{tabular}{c|l|l|l}
$\begin{array}{c}\text { Sector } \\
\text { ID }\end{array}$ & \multicolumn{1}{|c|}{$\begin{array}{c}\text { Pre-amp } \\
\text { design }\end{array}$} & $\begin{array}{c}\text { Reset } \\
\text { type }\end{array}$ & \multicolumn{1}{|c}{$\begin{array}{c}\text { Implant } \\
\text { configuration }\end{array}$} \\
\hline o & $\begin{array}{l}\text { enlarged } \\
\text { transistor FE }\end{array}$ & $\begin{array}{l}\text { diode } \\
\text { reset }\end{array}$ & $\begin{array}{l}\text { continuous n- } \\
\text { layer }\end{array}$ \\
\hline 1 & $\begin{array}{l}\text { enlarged } \\
\text { transistor FE }\end{array}$ & $\begin{array}{l}\text { diode } \\
\text { reset }\end{array}$ & $\begin{array}{l}\text { extra deep P- } \\
\text { well }\end{array}$ \\
\hline 2 & $\begin{array}{l}\text { enlarged } \\
\text { transistor FE }\end{array}$ & $\begin{array}{l}\text { PMOS } \\
\text { reset }\end{array}$ & $\begin{array}{l}\text { continuous n- } \\
\text { layer }\end{array}$ \\
\hline 3 & $\begin{array}{l}\text { enlarged } \\
\text { transistor FE }\end{array}$ & $\begin{array}{l}\text { diode } \\
\text { reset }\end{array}$ & n- gap \\
\hline 4 & $\begin{array}{l}\text { standard } \\
\text { transistor FE }\end{array}$ & $\begin{array}{l}\text { diode } \\
\text { reset }\end{array}$ & $\begin{array}{l}\text { continuous n- } \\
\text { layer }\end{array}$ \\
\hline 5 & $\begin{array}{l}\text { standard } \\
\text { transistor FE }\end{array}$ & $\begin{array}{l}\text { diode } \\
\text { reset }\end{array}$ & $\begin{array}{l}\text { extra deep P- } \\
\text { well }\end{array}$ \\
\hline 6 & $\begin{array}{l}\text { standard } \\
\text { transistor FE }\end{array}$ & $\begin{array}{l}\text { PMOS } \\
\text { reset }\end{array}$ & $\begin{array}{l}\text { continuous n- } \\
\text { layer }\end{array}$ \\
\hline 7 & $\begin{array}{l}\text { standard } \\
\text { transistor FE }\end{array}$ & $\begin{array}{l}\text { diode } \\
\text { reset }\end{array}$ & n- gap \\
\hline
\end{tabular}

This configuration allows the direct comparison of old and new pixel designs within the same pixel matrix and the same chip sample. 


\section{TEST BEAM MEASUREMENTS}

Test beam measurements were performed in April 2019 at the DESY beam facility in Hamburg and the ELSA beam facility in Bonn. Since both campaigns were done simultaneously, different setups had to be used. In the following chapters, only the setup and measurements at ELSA are discussed [8].

Measurements were performed with a telescope made from 7 full-sized MALTA chips (prototypes of the generation previous to Mini-MALTA) and a $3 \mathrm{GeV}$ electron beam. The telescope alignment and tracking were done with the Proteus software package [9] using a General Broken Lines (GBL) tracking algorithm [10]. An image of the test beam setup is shown in Fig. 5. Due to the low energy beam and significant multiple scattering only the innermost planes of both telescope arms as well as the reference plane close to the DUT (device under test) were used for final tracking (planes 2,3 and REF in Fig. 6).

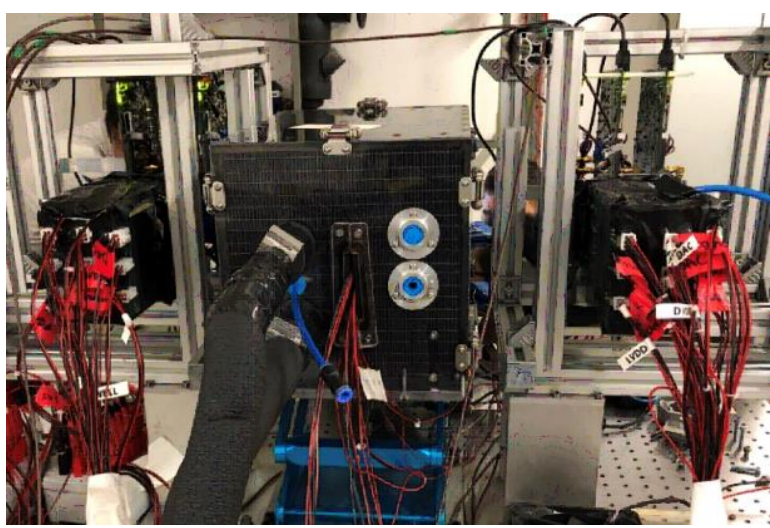

Figure 5. Image of the experimental setup at ELSA, Bonn. Both telescope arms contain 3 Malta planes. The cold box between the arms contains the DUT (Mini-Malta) and a reference plane (Malta) [8]

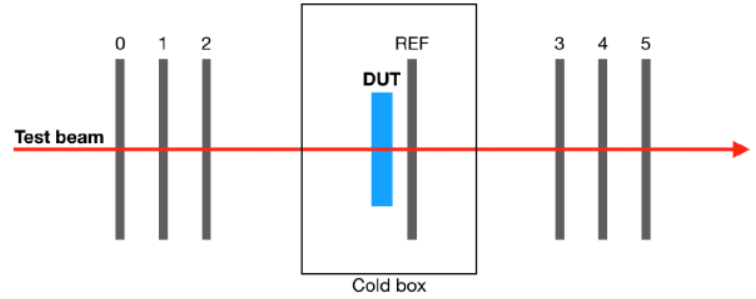

Figure 6. Geometry of telescope planes and DUT. Final tracking only included planes 2,3 and REF [8]

\section{DATA ANALYSIS}

Using a GBL algorithm for tracking, a 13.5 micron resolution was achieved at the position of the DUT (see Fig. 7). For efficiency calculations only tracks with $\mathrm{X}^{2} / \mathrm{DOF}<10$ (reduced chi-squared) were used and only tracks with and interpolated intersection on the DUT within 100 microns of the center of a pixel cluster were matched.

Due to the finite resolution of the telescope and the small dimensions of the pixel matrix (only $16 \times 64$ pixels) masked pixels reduced the efficiency of surrounding pixels and the overall efficiency of the matrix significantly. In order to account for this effect, a circle shaped masked region with a radius of 30 microns was put around every masked pixel. Tracks with an interpolated intersection inside such a region were not considered for efficiency calculations.
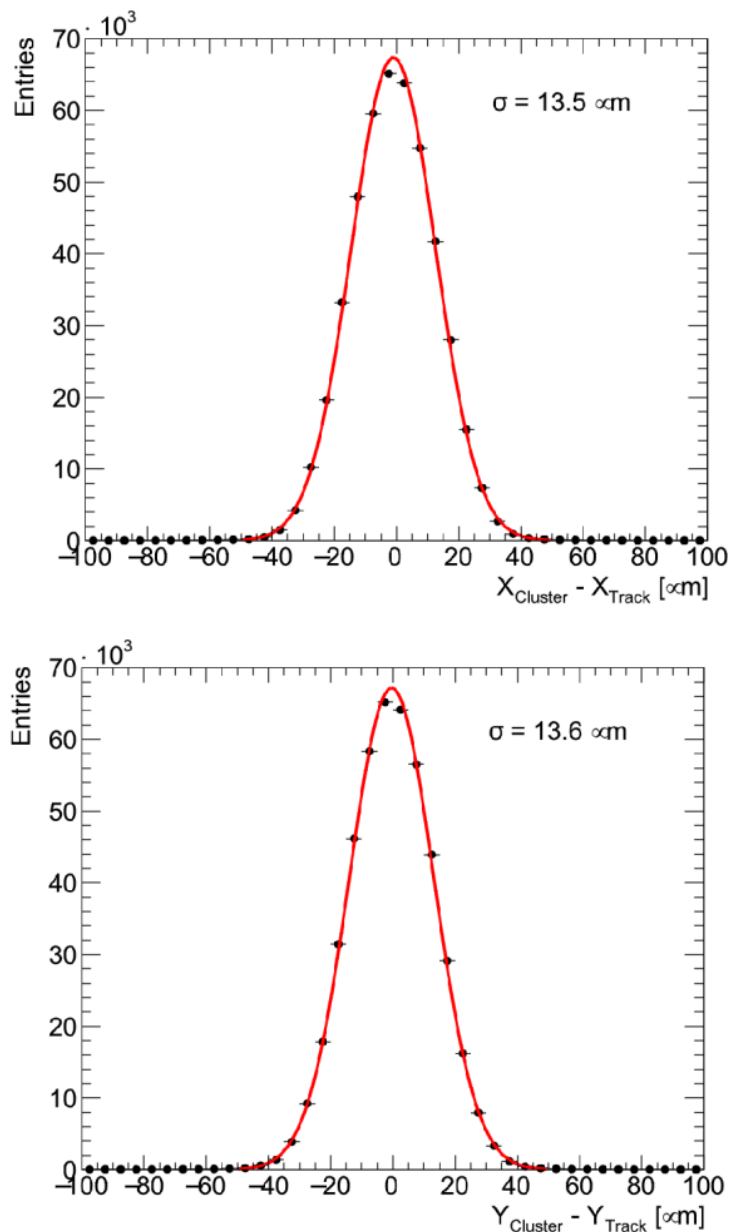

Figure 7. Telescope resolution at DUT position. Calculated as the sigma of the gaussian fit of the distribution of distances between matched clusters and tracks on the DUT [8]

\section{RESULTS}

The data recorded at the ELSA facility shows that the new pixel designs maintain almost full efficiency after irradiation to $10^{15} 1 \mathrm{MeV} \mathrm{n}$ eq $/ \mathrm{cm}^{2}$ which is equivalent to the lifetime dose at the $5^{\text {th }}$ layer of the new ATLAS ITk (Inner Tracker) which will be installed in 2025 .

A plot of the performance of the various sectors of Mini-MALTA after irradiation can be seen in Fig. 8. The sectors on the left feature an enlarged transistor analogue front end to improve the gain after irradiation. Counting from the bottom, the second and fourth sectors correspond to the extended p-well and n-gap pixel designs, respectively. The third sectors feature a PMOS reset instead of a diode reset and thus require a different chip configuration to operate. These sectors were not investigated. The threshold for the left sector was measured to be 200 electrons and was thus significantly lower than on the right side (340 electrons). The sectors with extended deep p-well and 
n-gap achieved an efficiency of $97.9 \%$ and $97.6 \%$, respectively.

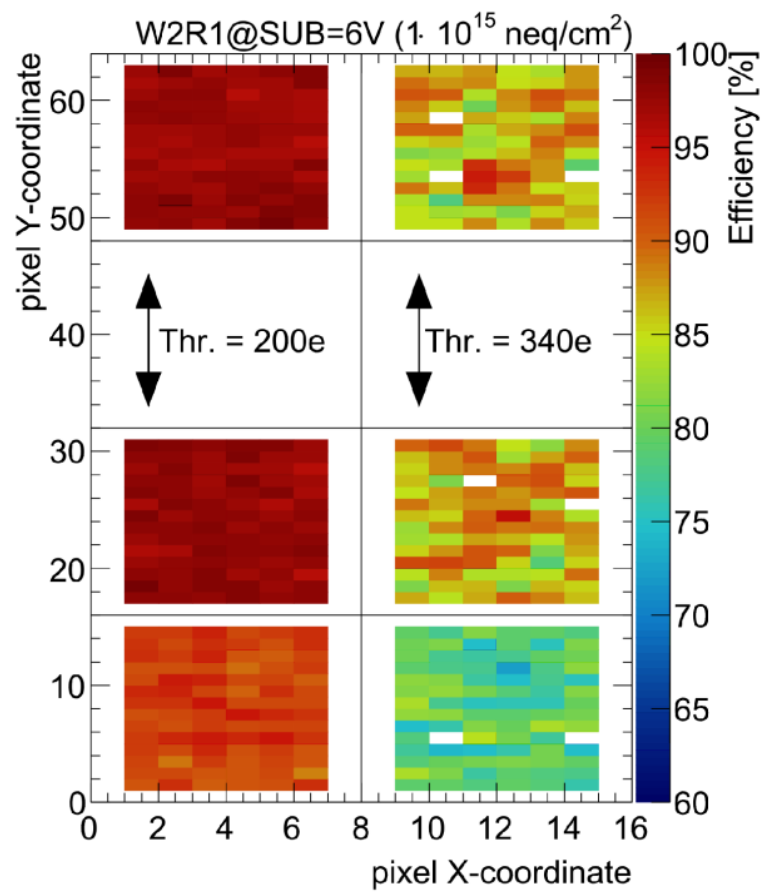

Figure 8. Efficiency of Mini-Malta after irradiation. On the left side (large transistors) the second and fourth sector correspond to the extended p-well and n-gap, respectively [8]

\section{CONCLUSION}

The CERN ATLAS Pixel group successfully developed a silicon CMOS DMAPS chip with the TowerJazz $180 \mathrm{~nm}$ process which shows sufficient radiation hardness for the $5^{\text {th }}$ layer of the ATLAS ITk, which will be installed in 2025 .

A new full-sized prototype with the new pixel design is currently being tested in the laboratory and beam tests at ELSA will be performed shortly.

Acknowledgements: Measurements leading to these results have been performed at the $E_{3}$ beam-line at the electron accelerator ELSA operated by the University of Bonn in Nordrhein-Westfalen, Germany.

This project has received funding from the European Union's Horizon 2020 Research and Innovation programme under Grant Agreement no. 654168 (IJS, Ljubljana, Slovenia).

\section{REFERENCES}

1. ATLAS Phase-II Upgrade Scoping Document, Rep. CERN-LHCC-2015-020; LHCC-G-166, CERN, Geneva, Switzerland, 2015.

Retrieved from: https://cds.cern.ch/record/2055248 Retrieved on: Feb. 25, 2019

2. Technical Design Report for the ATLAS Inner Tracker Pixel Detector, Rep. CERN-LHCC-2017-021; ATLASTDR-030, CERN, Geneva, Switzerland, 2017. Retrieved from: https://cds.cern.ch/record/2285585 Retrieved on: Feb. 25, 2019

3. P.S. Miyagawa, I. Dawson, Radiation background studies for the Phase II inner tracker upgrade, Rep. ATLUPGRADE-PUB-2013-012, CERN, Geneva, Switzerland, 2013.

Retrieved from: https://cds.cern.ch/record/1516824 Retrieved on: Feb. 25, 2019

4. H. Pernegger et al., "First tests of a novel radiation hard CMOS sensor process for Depleted Monolithic Active Pixel Sensors," J. Instrum., vol. 12, no. 6, Jun. 2017. DOI: $10.1088 / 1748-0221 / 12 / 06 /$ Po6008

5. I. Caicedo et al., "The Monopix chips: Depleted monolithic active pixel sensors with a column-drain read-out architecture for the ATLAS Inner Tracker upgrade," in Proc. 9th Int. Workshop Semicond. Pixel Detect. Part. Imaging (PIXEL 2018), Taipei, Taiwan, 2018.

DOI: 10.1088/1748-0221/14/06/Co6006

6. R. Cardella et al., "MALTA: an asynchronous readout CMOS monolithic pixel detector for the ATLAS HighLuminosity upgrade," in Proc. 9th Int. Workshop Semicond. Pixel Detect. Part. Imaging (PIXEL 2018), Taipei, Taiwan, 2018 DOI : 10.1088/1748-0221/14/06/Co6019

7. M. Munker et al., "Simulations of CMOS pixel sensors with a small collection electrode, improved for a faster charge collection and increased radiation tolerance," J. Instrum., vol. 14, no. 5, May 2019. DOI: 10.1088/1748-0221/14/05/C05013

8. I. A. Tortajada, "MiniMALTA: Radiation hard pixel designs for small-electrode monolithic CMOS sensors for the High Luminosity LHC," J. Instrum., Unpublished.

9. M. Kiehn et al., Proteus beam telescope reconstruction version 1.4.o, Zenodo, Geneva, Switzerland, 2019. DOI: 10.5281 /zenodo.2586736

10. C. Kleinwort, "General broken lines as advanced track fitting method," Nucl. Instrum. Methods Phys. Res. vol. 673, pp. 107 - 110, May 2012. DOI: 10.1016/j.nima.2012.01.024 